\title{
Design and Syntheses of Novel Phthalazin-1(2H)-one Derivatives as Acetohydroxyacid Synthase Inhibitors
}

\author{
Yuan-Xiang Li, ${ }^{1}$ Yan-Ping Luo, ${ }^{1}$ Zhen Xi, ${ }^{2, *}$ Congwei Niu, ${ }^{2}$ Yan-Zhen He, ${ }^{1}$ Guang-Fu Yang ${ }^{1, *}$ \\ ${ }^{1}$ Key Laboratory of Pesticide \& Chemical Biology, Ministry of Education, College of Chemistry, Central China \\ Normal University, Wuhan, Hubei 430079, P.R.China; ${ }^{2}$ State Key Laboratory of Elemento-Organic Chemistry, \\ Nankai University, Tianjin 300071, P. R. China
}

*To whom correspondence should be addressed. Fax: 86-27-67867141, E-mail: gfyang@mail.ccnu.edu.cn

Preparation of 1-(3-(benzyloxy) phenyl) ethanone (2). In a $250 \mathrm{~mL}$ two-neck round-bottom flask, $27.2 \mathrm{~g}(0.2 \mathrm{~mol})$ 3-hydroxyacetophenone (1), acetonitrile $(150 \mathrm{~mL})$ and $35.8 \mathrm{~g}(0.26 \mathrm{~mol})$ anhydrous $\mathrm{K}_{2} \mathrm{CO}_{3}$ were added gradually. The reaction was refluxed for $1 \mathrm{~h}$, benzyl bromide37.6 $\mathrm{g}$ (0.22 mol) was dropwise added during $30 \mathrm{~min}$, stirring for $3 \mathrm{~h}$ at refluxing. After cooling to room temperature, the precipitate was filtered off and washed with acetonitrile $50 \mathrm{~mL}$. The combined acetonitrile solutions were concentrated on a rotary evaporator to give crude 2 in yield $91 \%$ (41 g). Without purification the product was used directly for next reaction. ${ }^{1} \mathrm{H}$ NMR $\left(\mathrm{CDCl}_{3}\right): \delta 2.59$ (s, 3H, $\left.\mathrm{CH}_{3}\right), 5.11$ (s, 2H, $\left.\mathrm{CH}_{2}\right), 7.17-7.58$ (m, 9H, Ar-H).

Preparation of 2-(3-(benzyloxy)phenyl)-2-methyl-1,3-dioxolane (3). To $10.0 \mathrm{~g}$ (0.044 mol) 1-(3-(benzyloxy)phenyl)ethanone (2), $27.53 \mathrm{~g}(0.44 \mathrm{~mol})$ of ethylene glycol $39.5 \mathrm{~g}(0.064 \mathrm{~mol})$ of triethoxymethane and $0.46 \mathrm{~g}$ ( $2.4 \mathrm{mmol})$ of 4-methylbenzenesulfonic acid placed in a $100 \mathrm{~mL}$ two neck round-bottom flask were added gradually. The reaction was refluxed in oil bath. The course of reaction was observed by TLC. The resulting mixture was cooled to room temperature and was 
extracted with toluene $(50 \mathrm{~mL} \times 3)$, washed with saturated sodium hydrogen carbonate $(200 \mathrm{~mL})$ and water $(300 \mathrm{~mL})$, dried with anhydrous magnesium sulfate, filtered off with suction. The combined organic layer were concentrated on a rotary evaporator to give crude 10.93 g, which was purified by chromatography on silica using petroleum ether/ether (40:1) as eluent to give colorless oil 3 in yield 75\% (8.2 g). ${ }^{1} \mathrm{H}$ NMR $\left(\mathrm{CDCl}_{3}\right): \delta 1.65$ (s, 3H, $\left.\mathrm{CH}_{3}\right), 3.76$ (m, 2H, $\left.\mathrm{CH}_{2}\right), 4.02\left(\mathrm{~m}, 2 \mathrm{H}, \mathrm{CH}_{2}\right), 5.07$ (s, 2H, $\mathrm{BnCH}_{2}$ ), 6.89-7.45 (m, 9H, Ar-H).

Preparation of methyl 2-(benzyloxy)-6-(2-methyl-1,3-dioxolan-2-yl)benzoate (4). To 3.3 g (12.2 mmol) 2-(3-(benzyloxy)phenyl)-2-methyl-1,3-dioxolane (3) and $25 \mathrm{~mL}$ of toluene placed in a $50 \mathrm{ml}$ three neck round-bottom flask were added gradually. N-Butyllithium-hexane solution (2.2 M, $7 \mathrm{~mL}$, 0.015 mol) was dropwise added below $-8{ }^{\circ} \mathrm{C}$ (ice salt bath) during 10 min under $\mathrm{N}_{2}$ flow. The mixture was stirred at ambient temperature for $3 \mathrm{~h}$, and cooled to $-8{ }^{\circ} \mathrm{C}$. Methyl chloroformate 1.44 g (14.6 mmol) was added over $10 \mathrm{~min}$ to the resulting mixture, which was then stirred at room temperature overnight and poured into water $(200 \mathrm{~mL})$, extracted with toluene $(50 \mathrm{~mL} \times 3)$, washed with saturated sodium chloride $(150 \mathrm{~mL} \times 3)$, dried with anhydrous magnesium sulfate, filtered off with suction. The combined organic layer were concentrated on a rotary evaporator to give yellow solid $4.3 \mathrm{~g}$, which was triturated with isopropyl ether (IPE) + hexane (2: 1 by volume, $12 \mathrm{~mL}$ ), white solid was separated, filtrated and dried with vacuum to give 4 in yield 50\% (2.01 g). m.p. 94-96 ${ }^{\circ} \mathrm{C}$ [ref (4), 95-96 ${ }^{\circ} \mathrm{C}$ ]. ${ }^{1} \mathrm{H}$ NMR $\left(\mathrm{CDCl}_{3}\right): \delta 1.65$ (s, 3H, $\left.\mathrm{CH}_{3}\right), 3.76\left(\mathrm{~m}, 2 \mathrm{H}, \mathrm{CH}_{2}\right), 3.88(\mathrm{~s}, 3 \mathrm{H}$, $\left.\mathrm{COOCH}_{3}\right), 4.02$ (m, 2H, $\mathrm{CH}_{2}$ ), 5.07 (s, 2H, $\mathrm{BnCH}_{2}$ ), 6.89-7.45 (m, 8H, Ar-H).

Preparation of methyl 2-hydroxy-6-(2-methyl-1,3-dioxolan-2-yl)benzoate (5). To 1.0 g (3 mmol) methyl 2-(benzyloxy)-6-(2-methyl-1,3-dioxolan-2-yl)benzoate (4), 10 \% Pd/C 0.12 g and 15 mL of ethyl acetate placed in a $50 \mathrm{~mL}$ two neck round-bottom flask were added gradually. The mixture 
was stirred at room temperature under $\mathrm{H}_{2}$ flow until the material was completely transformed by TLC. The resulting mixture was filtered off with suction, concentrated on a rotary evaporator to give white solid 5 in 94\% (0.68 g). m.p. $141-143{ }^{\circ} \mathrm{C}$ [ref (4), 142-143 ${ }^{\circ} \mathrm{C}$ ]. ${ }^{1} \mathrm{H}$ NMR $\left(\mathrm{CDCl}_{3}\right): \delta 1.84$ (s, 3H, $\mathrm{CH}_{3}$ ), 3.56 (m, 2H, $\mathrm{CH}_{2}$ ), 3.91 (s, 3H, $\mathrm{COOCH}_{3}$ ), 3.94 (m, 2H, $\mathrm{CH}_{2}$ ), 6.91 (d, $J=8.0 \mathrm{~Hz}, 1 \mathrm{H}$, Ar-H), 7.14 (d, $J=8.0 \mathrm{~Hz}, 1 \mathrm{H}, \mathrm{Ar}-\mathrm{H}), 7.28$ (t, $J=8.0 \mathrm{~Hz}, 1 \mathrm{H}, \mathrm{Ar}-\mathrm{H}), 7.48$ (s, 1H, OH).

\section{Preparation of methyl 2-(4,6-dimethoxypyrimidin-2-yloxy)-6-(2-methyl-1,3-dioxolan-2-yl)}

benzoate (6). To $0.51 \mathrm{~g}$ (2.1 mmol) methyl 2-hydroxy-6-(2-methyl-1,3-dioxolan-2-yl)benzoate (5) and anhydrous $\mathrm{K}_{2} \mathrm{CO}_{3} 0.59 \mathrm{~g}(4.2 \mathrm{mmol})$ in acetone $(10 \mathrm{~mL})$ was stirred at $56{ }^{\circ} \mathrm{C}$ for $1 \mathrm{~h}$. 4,6-dimethoxy-2-(methylsulfonyl)pyrimidine (DMSP) $0.49 \mathrm{~g}$ (2.25 mmol) was added. The reaction was continued for $10 \mathrm{~h}$. The resulting mixture was cooled to room temperature, filtered with infusorial earth and concentrated on a rotary evaporator to give crude white solid, crystalline 6 could be obtained by recrystallization from ethanol. Yield 80\%, m.p. 131-133 ${ }^{\circ} \mathrm{C} .{ }^{1} \mathrm{H} \mathrm{NMR}\left(\mathrm{CDCl}_{3}\right)$ : $\delta 1.76\left(\mathrm{~s}, 3 \mathrm{H}, \mathrm{CH}_{3}\right), 3.68\left(\mathrm{~m}, 2 \mathrm{H}, \mathrm{CH}_{2}\right), 3.78\left(\mathrm{~s}, 3 \mathrm{H}, \mathrm{COOCH}_{3}\right), 3.90\left(\mathrm{~s}, 6 \mathrm{H}, \mathrm{OCH}_{3} \times 2\right), 3.97(\mathrm{~m}$, 2H, $\mathrm{CH}_{2}$ ), 5.75 (s, 1H, PyHet-H), 7.16-7.40 (m, 3H, Ar-H).

Data for 7c. Yield, 82\%; mp,108-110 ${ }^{\circ} \mathrm{C} .{ }^{1} \mathrm{H}$ NMR (400 MHz, $\left.\mathrm{CDCl}_{3}\right) \delta: 1.23$ (t, 3H, $\mathrm{OCH}_{2} \mathrm{CH}_{3}$ ), 2.59 (s, 3H, $\left.\mathrm{CH}_{3}\right), 3.76$ (s, 6H, $\left.2 \times \mathrm{OCH}_{3}\right), 4.16-4.18\left(\mathrm{~m}, 2 \mathrm{H}, \mathrm{OCH}_{2} \mathrm{CH}_{3}\right), 4.81\left(\mathrm{~s}, 2 \mathrm{H}, \mathrm{CH}_{2}\right), 5.74$ (s, 1H, PyHet-H), 7.51 (d, $J=8.0$ Hz, 1H,7`-ArH), 7.68 (d, $J=8.0 \mathrm{~Hz}, 1 \mathrm{H}, 5 `-\mathrm{ArH}), 7.85$ (t, $J=8.0 \mathrm{~Hz}$, 1H, 6 -ArH). EI MS: m/z (\%) 400 (M+1, 83), 369 (46), 329 (90), 327 (100), 301 (33), 299 (30), 295 (30), 267 (25), 255 (27), 229 (15). Anal. Calcd for $\mathrm{C}_{19} \mathrm{H}_{20} \mathrm{~N}_{4} \mathrm{O}_{6}$ : C, 57.00; H, 5.03; N, 13.99. Found: C, 56.26; H, 5.31; N, 13.97.

Data for 7d. Yield, 81\%; mp 166-168 ${ }^{\circ} \mathrm{C} .{ }^{1} \mathrm{H}$ NMR (400 MHz, $\left.\mathrm{CDCl}_{3}\right): \delta 1.29(\mathrm{t}, J=14.4 \mathrm{~Hz}, 3 \mathrm{H}$, $\mathrm{CH}_{2} \mathrm{CH}_{3}$ ), 2.59 (s, 3H, $\mathrm{CH}_{3}$ ), 3.77 (s, $6 \mathrm{H}, 2 \times \mathrm{OCH}_{3}$ ), 4.12-4.13 (dd, $J=7.2 \mathrm{~Hz}, 2 \mathrm{H}, \mathrm{CH}_{2} \mathrm{CH}_{3}$ ), 5.75 
(s, 1H, PyHet-H), 7.49 (d, $\left.J=8.0 \mathrm{~Hz}, 1 \mathrm{H}, 7^{`}-\mathrm{ArH}\right), 7.65$ (d, $\left.J=8.0 \mathrm{~Hz}, 1 \mathrm{H}, 5^{`}-\mathrm{ArH}\right), 7.82$ (t, $J=$ $\left.8.0 \mathrm{~Hz}, 1 \mathrm{H}, 6^{`}-\mathrm{ArH}\right)$. EI MS: m/z (\%) 342 (M+1 100), 327 (22), 315 (23), 311 (69), 285 (32), 283 (35), 256 (28). Anal. Calcd for $\mathrm{C}_{17} \mathrm{H}_{18} \mathrm{~N}_{4} \mathrm{O}_{4}$ : C, 59.64; H, 5.30; N, 16.37C. Found: C, 59.42; H, 5.51; N, 16.12.

Data for 7e. Yield, 93\%; mp 167-168 ${ }^{\circ} \mathrm{C} .{ }^{1} \mathrm{H}$ NMR (400 MHz, $\left.\mathrm{CDCl}_{3}\right): \delta=2.57$ (s, 3H, $\left.\mathrm{CH}_{3}\right), 3.70$ (s, 6H, 2× $\mathrm{OCH}_{3}$ ), 5.25 (s, 2H, $\mathrm{CH}_{2}$ ), 5.75 (s, 1H, PyHet-H), 7.23-7.81 (m, 8H, 8ph-H). EI MS: $m / z$ (\%) 404 (M+, 74$), 300$ (100), 284 (88), 258 (41), 256 (89), 230 (34), 91 (56). Anal. Calcd for $\mathrm{C}_{22} \mathrm{H}_{20} \mathrm{~N}_{4} \mathrm{O}_{4}$ : C, 65.34; H, 4.98; N, 13.85. Found: C, 65.08; H, 5.17; N, 13.70.

Data for 7f. Yield, 92\%; mp 149-151 ${ }^{\circ} \mathrm{C} .{ }^{1} \mathrm{H}$ NMR (400 MHz, $\mathrm{CDCl}_{3}$ ): $\delta 2.57$ (s, 3H, $\mathrm{CH}_{3}$ ), 3.74 (s, 6H, 2× $\mathrm{OCH}_{3}$ ), 5.27 (s, 2H, $\mathrm{CH}_{2}$ ), 5.75 (s, 1H, PyHet-H), 7.02-7.846 (m, 63H, 6ph-H). EI MS: $m / z$ (\%) 502 ([M+1] $\left.]^{+}, 13\right), 500$ ([M-1] $\left.]^{+}, 12\right), 300$ (62), 284 (84), 258 (49), 256 (100), 230 (71), 187 (86), 139 (42), 108 (42). Anal. Calcd for $\mathrm{C}_{22} \mathrm{H}_{18} \mathrm{BrFN}_{4} \mathrm{O}_{4}$ : C, 52.71; H, 3.62; N, 11.18. Found: C, 52.61; H, 3.80; N, 11.01.

Data for 7g. Yield, 86\%; mp 171-173 ${ }^{\circ} \mathrm{C} .{ }^{1} \mathrm{H}$ NMR (400 MHz, $\left.\mathrm{CDCl}_{3}\right): \delta 2.50$ (s, 3H, $\mathrm{CH}_{3}$ ), 3.75 (s, 6H, 2× $\mathrm{OCH}_{3}$ ), 5.35 (s, 2H, $\mathrm{CH}_{2}$ ), 5.74 (s, 1H, PyHet-H), 6.82-7.81 (m, 6H, 6ph-H). EI MS: m/z (\%) $441\left([\mathrm{M}+1]^{+}, 7\right), 440\left(\mathrm{M}^{+}, 39\right), 409$ (6), 300 (29), 284 (65), 258 (62), 256 (89), 230 (70), 138 (25), 127 (100). Anal. Calcd for $\mathrm{C}_{22} \mathrm{H}_{18} \mathrm{~F}_{2} \mathrm{~N}_{4} \mathrm{O}_{4}$ : C, 60.00; H, 4.12; N, 12.72. Found: C, 59.96; H, 4.34; N, 12.49.

Data for $7 \boldsymbol{h}$. Yield, 87\%; mp 155-157 ${ }^{\circ} \mathrm{C} .{ }^{1} \mathrm{H}$ NMR (400 MHz, $\mathrm{CDCl}_{3}$ ): $\delta 2.57$ (s, 3H, $\mathrm{CH}_{3}$ ), 3.74 (s, 6H, $2 \times \mathrm{OCH}_{3}$ ), 5.28 (s, 2H, $\mathrm{CH}_{2}$ ), 5.75 (s, 1H, PyHet-H), 6.71-7.86 (m, 6H, 6ph-H). EI MS: m/z (\%) 441 ([M+1] $\left.]^{+}, 7\right), 440\left(\mathrm{M}^{+}, 31\right), 409$ (1), 300 (45), 284 (83), 258 (45), 256 (100), 230 (55), 138 (18), 127 (88). Anal. Calcd for $\mathrm{C}_{22} \mathrm{H}_{18} \mathrm{~F}_{2} \mathrm{~N}_{4} \mathrm{O}_{4}$ : C, 60.00; H, 4.12; N, 12.72. Found: C, 59.75; H, 4.35; N, 
12.53.

Data for 7i. Yield, 78\%; mp 163-164 ${ }^{\circ} \mathrm{C} .{ }^{1} \mathrm{H}$ NMR (400 MHz, $\mathrm{CDCl}_{3}$ ): $\delta 2.58$ (s, 3H, $\mathrm{CH}_{3}$ ), 3.72 (s, 6H, $2 \times \mathrm{OCH}_{3}$ ), 5.24 (s, 2H, $\mathrm{CH}_{2}$ ), 5.75 (s, 1H, PyHet-H), 6.90-7.85 (m, 7H, 7ph-H). EI MS: m/z (\%) 423 ([M+1] $\left.]^{+}, 8\right), 422\left(\mathrm{M}^{+}, 37\right), 300$ (57), 284 (98), 258 (50), 256 (95), 230 (53), 228 (21), 200 (14), 138 (40), 109 (100) . Anal. Calcd for $\mathrm{C}_{22} \mathrm{H}_{19} \mathrm{FN}_{4} \mathrm{O}_{4}$ : C, 62.55; H, 4.53; N, 13.26. Found: C, 62.57; H, 4.79; N, 13.02.

Data for 7j. Yield, 59\%; mp 163-164 ${ }^{\circ} \mathrm{C} .{ }^{1} \mathrm{H}$ NMR (400 MHz, $\mathrm{CDCl}_{3}$ ): $\delta 2.58$ (s, 3H, $\mathrm{CH}_{3}$ ), 3.76 (s, 6H, $2 \times \mathrm{OCH}_{3}$ ), 5.36 (s, 2H, $\mathrm{CH}_{2}$ ), 5.73 (s, 1H, PyHet-H), 6.83-7.86 (m, 7H, 7ph-H). EI MS: m/z (\%) 485([M+2] $\left.]^{+}, 2\right), 483\left(\mathrm{M}^{+}, 2\right), 403$ (76), 297 (9), 249 (24), 171 (96), 169 (100), 139 (37), 89 (66). Anal. Calcd for $\mathrm{C}_{22} \mathrm{H}_{19} \mathrm{BrN}_{4} \mathrm{O}_{4}$ : C, 54.67; H, 3.96; N, 11.59. Found: C, 54.58; H, 4.21; N, 11.31.

Data for 7k. Yield, 97\%; mp 165-167 ${ }^{\circ} \mathrm{C} .{ }^{1} \mathrm{H}$ NMR (400 MHz, $\left.\mathrm{CDCl}_{3}\right): \delta 2.58$ (s, 3H, $\mathrm{CH}_{3}$ ), 3.72 (s, 6H, $2 \times \mathrm{OCH}_{3}$ ), 5.21 (s, 2H, $\mathrm{CH}_{2}$ ), 5.76 (s, 1H, PyHet-H), 7.11-7.85 (m, 7H, 7ph-H). EI MS: m/z (\%) $484\left([\mathrm{M}+1]^{+}, 41\right), 483\left(\mathrm{M}^{+}, 11\right), 482$ ([M-1] $\left.]^{+}, 21\right), 403$ (6), 343 (20), 300 (100), 285 (28), 284 (99), 271 (23), 258 (57), 256 (60), 230 (72), 171 (43), 169 (51), 138 (37). Anal. Calcd for $\mathrm{C}_{22} \mathrm{H}_{19} \mathrm{BrN}_{4} \mathrm{O}_{4}$ : C, 54.67; H, 3.96; N, 11.59. Found: C, 54.97; H, 4.20; N, 11.54.

Data for 7l. Yield, 52\%; mp 191-193 ${ }^{\circ} \mathrm{C} .{ }^{1} \mathrm{H}$ NMR (400 MHz, $\mathrm{CDCl}_{3}$ ): $\delta 2.57$ (s, 3H, $\mathrm{CH}_{3}$ ), 3.71 (s, 6H, $2 \times \mathrm{OCH}_{3}$ ), 5.19 (s, 2H, CH 2 ), 5.76 (s, 1H, PyHet-H), 7.20-7.82 (m, 7H, 7ph-H). EI MS: m/z (\%) $484\left([\mathrm{M}+1]^{+}, 41\right), 483\left(\mathrm{M}^{+}, 11\right), 482$ ([M-1] $\left.]^{+}, 6\right), 343$ (11), 300 (86), 284 (68), 271 (13), 258 (67), 256 (100), 230 (58), 171 (56), 169 (54), 138 (29). Anal. Calcd for $\mathrm{C}_{22} \mathrm{H}_{19} \mathrm{BrN}_{4} \mathrm{O}_{4}$ : C, 54.67; H, 3.96; N, 11.59. Found: C, 54.74; H, 4.19; N, 11.40.

Data for $7 \boldsymbol{m}$. Yield, 85\%; mp 168-170 ${ }^{\circ} \mathrm{C} .{ }^{1} \mathrm{H}$ NMR (400 MHz, $\mathrm{CDCl}_{3}$ ): $\delta 2.30$ (s, 3H, ph-CH 3 ), 2.56 (s, 3H, $\mathrm{CH}_{3}$ ), 3.71 (s, 6H, 2x $\mathrm{OCH}_{3}$ ), 5.21 (s, 2H, $\mathrm{CH}_{2}$ ), 5.76 (s, 1H, PyHet-H), 7.05-7.80 (m, 
7H, 7ph-H). EI MS: m/z (\%) 419 ([M+1] $\left.]^{+}, 8\right), 418$ (M+, 34), 300 (86), 284 (97), 258 (93), 256 (100), 230 (68), 201 (21), 139 (35), 105 (99). Anal. calcd for $\mathrm{C}_{23} \mathrm{H}_{22} \mathrm{~N}_{4} \mathrm{O}_{4}$ : C, 66.02; H, 5.30; N, 13.39. Found: C, 66.35; H, 5.54; N, 13.24.

Data for 7n. Yield, 61\%; mp 186-188 ${ }^{\circ} \mathrm{C} .{ }^{1} \mathrm{H}$ NMR (400 MHz, $\left.\mathrm{CDCl}_{3}\right): \delta 2.29$ (s, 3H,), 2.57 (s, 3H, $\mathrm{CH}_{3}$ ), 3.71 (s, 6H, 2x $\mathrm{OCH}_{3}$ ), 5.21 (s, 2H, $\mathrm{CH}_{2}$ ), 5.76 (s, 1H, PyHet-H), 7.21-7.83 (m, 7H, 7ph-H).

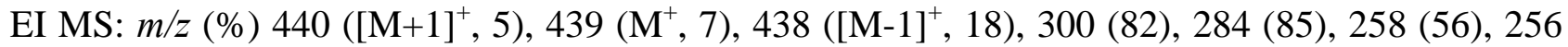
(100), 230 (53), 125 (46). Anal. Calcd for $\mathrm{C}_{22} \mathrm{H}_{19} \mathrm{ClN}_{4} \mathrm{O}_{4}$ : C, 60.21; H, 4.36; Cl, 8.08; N, 12.77. Found: C, 60.09; H, 4.64; N, 12.53.

Data for 7o. Yield, 65\%; mp 181-183 ${ }^{\circ} \mathrm{C} .{ }^{1} \mathrm{H}$ NMR (400 MHz, $\mathrm{CDCl}_{3}$ ): $\delta=2.30$ (s, 3H, ph-CH$)_{3}$, 2.58 (s, 3H, $\mathrm{CH}_{3}$ ), 3.77 (s, 6H, 2× $\mathrm{OCH}_{3}$ ), 5.66 (s, 2H, $\mathrm{CH}_{2}$ ), 5.75 (s, 1H, PyHet-H), 6.80-7.99 (m, 6H, 6ph-H). EI MS: m/z (\%) 463 (M+1 4 ), 417 (61), 315 (34), 299 (24), 283 (73), 272 (19), 226 (22), 189 (28), 173 (100), 139 (81), 104 (65), 91 (95). Anal. Calcd for $\mathrm{C}_{23} \mathrm{H}_{21} \mathrm{~N}_{5} \mathrm{O}_{6}$ : C, 59.61; H, 4.57; N, 15.11. Found: C, 59.60; H, 4.80; N, 14.88.

Data for 7p. Yield, 93\%; mp 186-188 ${ }^{\circ} \mathrm{C} .{ }^{1} \mathrm{H}$ NMR (400 MHz, $\left.\mathrm{CDCl}_{3}\right): \delta=2.58\left(\mathrm{~s}, 3 \mathrm{H}, \mathrm{CH}_{3}\right), 3.76$ (s, 6H, 2x $\mathrm{OCH}_{3}$ ), 5.39 (s, 2H, $\mathrm{CH}_{2}$ ), 5.73 (s, 1H, PyHet-H), 6.89-7.87 (m, 7H, 7ph-H). EI MS: $m / z$ (\%) 439(M+, 2), 438 ([M-1] $\left.]^{+}, 1\right), 404$ (17), 403 (98), 300 (28), 284 (49), 258 (30), 256 (50), 230 (24), 139 (37), 125 (100), 89 (28). Anal. Calcd for $\mathrm{C}_{22} \mathrm{H}_{19} \mathrm{ClN}_{4} \mathrm{O}_{4}$ : C, 60.21; H, 4.36; N, 12.77. Found: C, 60.51; H, 4.63; N, 12.49.

Data for 7q. Yield, 89\%; mp $178 \sim 180{ }^{\circ} \mathrm{C} .{ }^{1} \mathrm{H}$ NMR (400 MHz, $\mathrm{CDCl}_{3}$ ): $\delta 2.59$ (s, 3H, $\mathrm{CH}_{3}$ ), 3.71 (s, 6H, 2× $\mathrm{OCH}_{3}$ ), 5.22 (s, 2H, $\mathrm{CH}_{2}$ ), 5.76 (s, 1H, PyHet-H), 7.20-7.84 (m, 7H, 7ph-H). EI MS: $m / z$ (\%) $\left.440\left([\mathrm{M}+1]^{+}, 6\right), 439\left(\mathrm{M}^{+}, 6\right), 438(\mathrm{M} \mathrm{-1})^{+}, 19\right), 300$ (57), 284 (57), 258 (41), 256 (90), 230 (45), 139 (47), 125 (100), 89 (29). Anal. Calcd for $\mathrm{C}_{22} \mathrm{H}_{19} \mathrm{ClN}_{4} \mathrm{O}_{4}$ : C, 60.21; H, 4.36; N, 12.77. Found: C, 
60.02; H, 4.61; N, 12.51.

Data for 7r. Yield, 77\%; mp 206-208 ${ }^{\circ} \mathrm{C} .{ }^{1} \mathrm{H}$ NMR (400 MHz, $\mathrm{CDCl}_{3}$ ): $\delta 2.36$ (s, 3H, ph-CH ), 2.56 (s, 3H, $\mathrm{CH}_{3}$ ), 3.73 (s, 6H, 2x $\mathrm{OCH}_{3}$ ), 5.28 (s, 2H, $\mathrm{CH}_{2}$ ), 5.74 (s, 1H, PyHet-H), 7.01-7.84 (m, 7H, 7ph-H). EI MS: m/z (\%) 419 ([M+1] $\left.]^{+}, 9\right), 418$ (M+, 37), 403 (17), 300 (100), 284 (76), 258 (46), 256 (64), 230 (35), 139 (28), 105 (49). Anal. Calcd for $\mathrm{C}_{23} \mathrm{H}_{22} \mathrm{~N}_{4} \mathrm{O}_{4}$ : C, 66.02; H, 5.30; N, 13.39. Found: C, 65.96; H, 5.58; N, 13.11.

Data for 7s. Yield, 93\%; mp 156-158 ${ }^{\circ} \mathrm{C} .{ }^{1} \mathrm{H}$ NMR (400 MHz, $\left.\mathrm{CDCl}_{3}\right): \delta 0.90(\mathrm{t}, J=7.2 \mathrm{~Hz}, 3 \mathrm{H}$, $\left.\left(\mathrm{CH}_{2}\right)_{3} \mathrm{CH}_{3}\right), 1.31\left(\mathrm{~m}, 2 \mathrm{H},\left(\mathrm{CH}_{2}\right)_{3} \mathrm{CH}_{3}\right), 1.71\left(\mathrm{~m}, 2 \mathrm{H},\left(\mathrm{CH}_{2}\right)_{3} \mathrm{CH}_{3}\right), 2.60$ (s, 3H, $\left.\mathrm{CH}_{3}\right), 3.77$ (s, 6H, 2 $\left.\times \mathrm{OCH}_{3}\right), 4.07\left(\mathrm{t}, J=7.6 \mathrm{~Hz}, 2 \mathrm{H},\left(\mathrm{CH}_{2}\right)_{3} \mathrm{CH}_{3}\right), 5.75$ (s, 1H, PyHet-H), 7.50-7.82 (m, 3H, 3ph-H). EI MS: $m / z(\%) 371$ ([M+1] $\left.]^{+}, 6\right), 370\left(\mathrm{M}^{+}, 16\right), 355$ (91), 313 (50), 299 (41), 283 (88), 256 (100), 230 (53), 214 (37), 189 (24), 173 (70), 139 (60). Anal. Calcd for $\mathrm{C}_{19} \mathrm{H}_{22} \mathrm{~N}_{4} \mathrm{O}_{4}$ : C, 61.61; H, 5.99; N, 15.13. Found: C, 64.56; H, 6.26; N, 14.88.

Data for 7t. Yield, 80\%; mp 129-131 ${ }^{\circ} \mathrm{C} .{ }^{1} \mathrm{H}$ NMR (400 MHz, $\left.\mathrm{CDCl}_{3}\right): \delta 0.90(\mathrm{t}, J=7.2 \mathrm{~Hz}, 3 \mathrm{H}$, $\left.\left(\mathrm{CH}_{2}\right)_{2} \mathrm{CH}_{3}\right), 1.75\left(\mathrm{~m}, 2 \mathrm{H},\left(\mathrm{CH}_{2}\right)_{2} \mathrm{CH}_{3}\right), 2.60\left(\mathrm{~s}, 3 \mathrm{H}, \mathrm{CH}_{3}\right), 3.77\left(\mathrm{~s}, 6 \mathrm{H}, 2 \times \mathrm{OCH}_{3}\right), 4.03(\mathrm{t}, J=7.6$ Hz, 2H, $\left.\left(\mathrm{CH}_{2}\right)_{2} \mathrm{CH}_{3}\right), 5.75$ (s, 1H, PyHet-H), 7.49-7.82 (m, 3H, 3ph-H). EI MS: m/z (\%) 357 ([M+1] $\left.]^{+}, 9\right), 356\left(\mathrm{M}^{+}, 31\right), 341$ (29), 314 (62), 283 (45), 256 (35), 230 (26), 200 (37), 188 (11), 172 (100). Anal. Calcd for $\mathrm{C}_{18} \mathrm{H}_{20} \mathrm{~N}_{4} \mathrm{O}_{4}$ : C, 60.66; H, 5.66; N, 15.72. Found: C, 60.49; H, 5.95; N, 15.42. Data for 7u. Yield, 69\%; mp 190-192 ${ }^{\circ} \mathrm{C} .{ }^{1} \mathrm{H}$ NMR (400 MHz, $\left.\mathrm{CDCl}_{3}\right): \delta 1.23$ (t, $J=7.6 \mathrm{~Hz}, 3 \mathrm{H}$, ph- $\mathrm{CH}_{2} \mathrm{CH}_{3}$ ), 2.66 (s, 3H, $\mathrm{CH}_{3}$ ), 2.67-2.68 (m, 2H, ph- $\mathrm{CH}_{2} \mathrm{CH}_{3}$ ), 3.77 (s, 6H, 2× $\mathrm{OCH}_{3}$ ), 5.72 (s, 1H, PyHet-H), 7.19-7.93 (m, 7H, 7ph-H). EI MS: m/z (\%) 446(M+, 2), 418 (7), 314 (2), 284 (3), 133 (100), 132 (30), 105 (19). Anal. Calcd for $\mathrm{C}_{24} \mathrm{H}_{22} \mathrm{~N}_{4} \mathrm{O}_{5}$ : C, 64.57; H, 4.97; N, 12.55. Found: C, 64.30; H, 5.21; N, 12.26 . 
Data for $7 v$. Yield, 81\%; mp 155-157 ${ }^{\circ} \mathrm{C} .{ }^{1} \mathrm{H}$ NMR (400 MHz, $\mathrm{CDCl}_{3}$ ): $\delta 1.42$ (s, 9H, $\left.\mathrm{COOC}_{4} \mathrm{H}_{9}-\mathrm{Bu}\right), 2.60$ (s, 3H, CH 3 ), 3.77 (s, $\left.6 \mathrm{H}, 2 \times \mathrm{OCH}_{3}\right), 4.71$ (s, $\left.2 \mathrm{H}, \mathrm{CH}_{2}\right), 5.72$ (s, 1H, PyHet-H), 7.51-7.85 (m, 3H, 3ph-H). EI MS: m/z (\%) 428 ( $\left.\mathrm{M}^{+}, 7\right), 355$ (13), 328 (68), 327 (100), 300 (25), 284 (30), 258 (20), 256 (44), 230 (24), 138 (18). Anal. Calcd for $\mathrm{C}_{21} \mathrm{H}_{24} \mathrm{~N}_{4} \mathrm{O}_{6}$ : C, 58.87; H, 5.65; N, 13.08. Found: C, 58.81; H, 5.94; N, 12.86.

Data for $7 w$. Yield, $41 \%$; mp 181-183 ${ }^{\circ} \mathrm{C} .{ }^{1} \mathrm{H}$ NMR $\left(400 \mathrm{MHz}, \mathrm{CDCl}_{3}\right)$ : $\delta 2.61$ (s, 3H, $\left.\mathrm{CH}_{3}\right), 3.77$ (s, 6H, $\left.2 \times \mathrm{OCH}_{3}\right), 3.86\left(\mathrm{~m}, 2 \mathrm{H},-\mathrm{OCH}_{2}\right), 4.00\left(\mathrm{~m}, 2 \mathrm{H},-\mathrm{OCH}_{2}\right), 4.24\left(\mathrm{~d}, J=5.2 \mathrm{~Hz}, 2 \mathrm{H}, \mathrm{N}-\mathrm{CH}_{2}\right), 5.34$ (t, $J=5.2 \mathrm{~Hz}, 1 \mathrm{H}, \mathrm{CH}), 5.72$ (s, 1H, PyHet-H), 7.49-7.85 (m, 3H, 3ph-H). EI MS: $m / z$ (\%) 401 ([M+1] $\left.]^{+}, 7\right), 400$ ( $\left.\mathrm{M}^{+}, 18\right), 372$ (16), 328 (71), 327 (26), 313 (40), 300 (26), 284 (40), 258 (31), 256 (70), 230 (39), 200 (24), 172 (36), 168 (38), 157 (26), 139 (36). Anal. Calcd for $\mathrm{C}_{19} \mathrm{H}_{20} \mathrm{~N}_{4} \mathrm{O}_{6}$ : C, 57.00; H, 5.03; N, 13.99. Found: C, 57.09; H, 5.30; N, 13.72. 

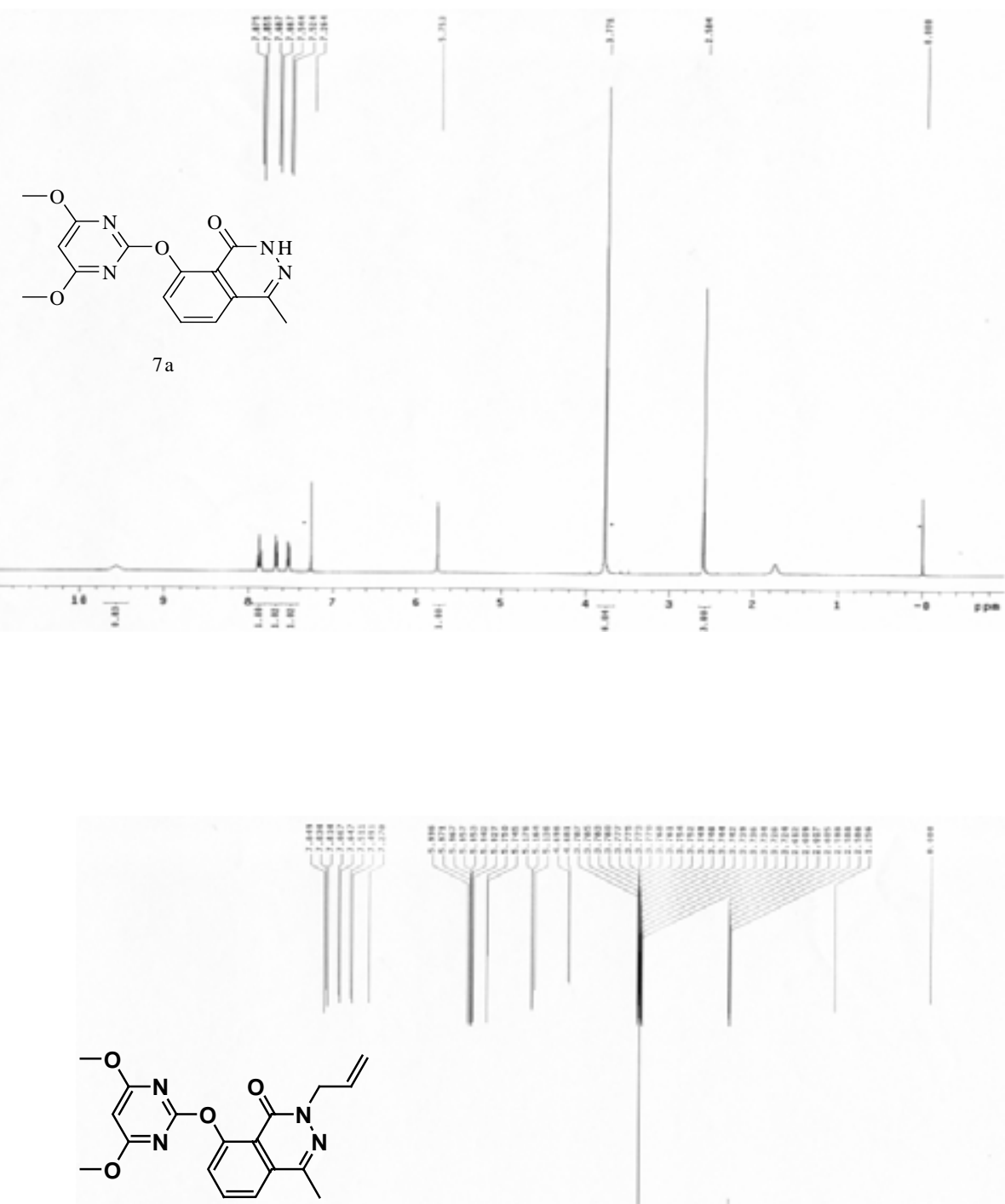

7b

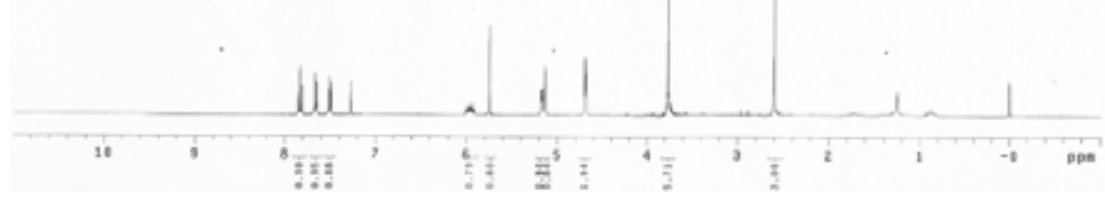



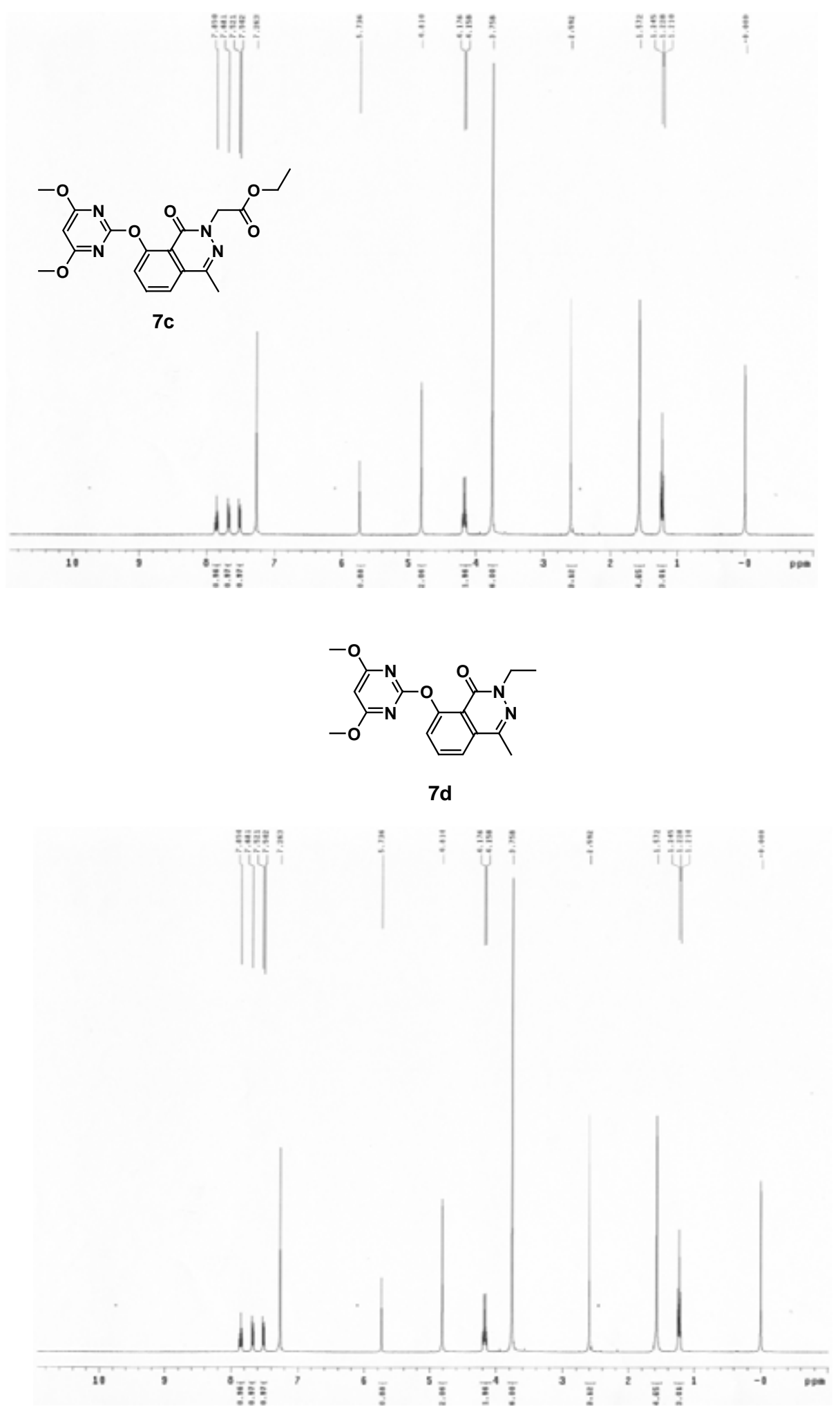

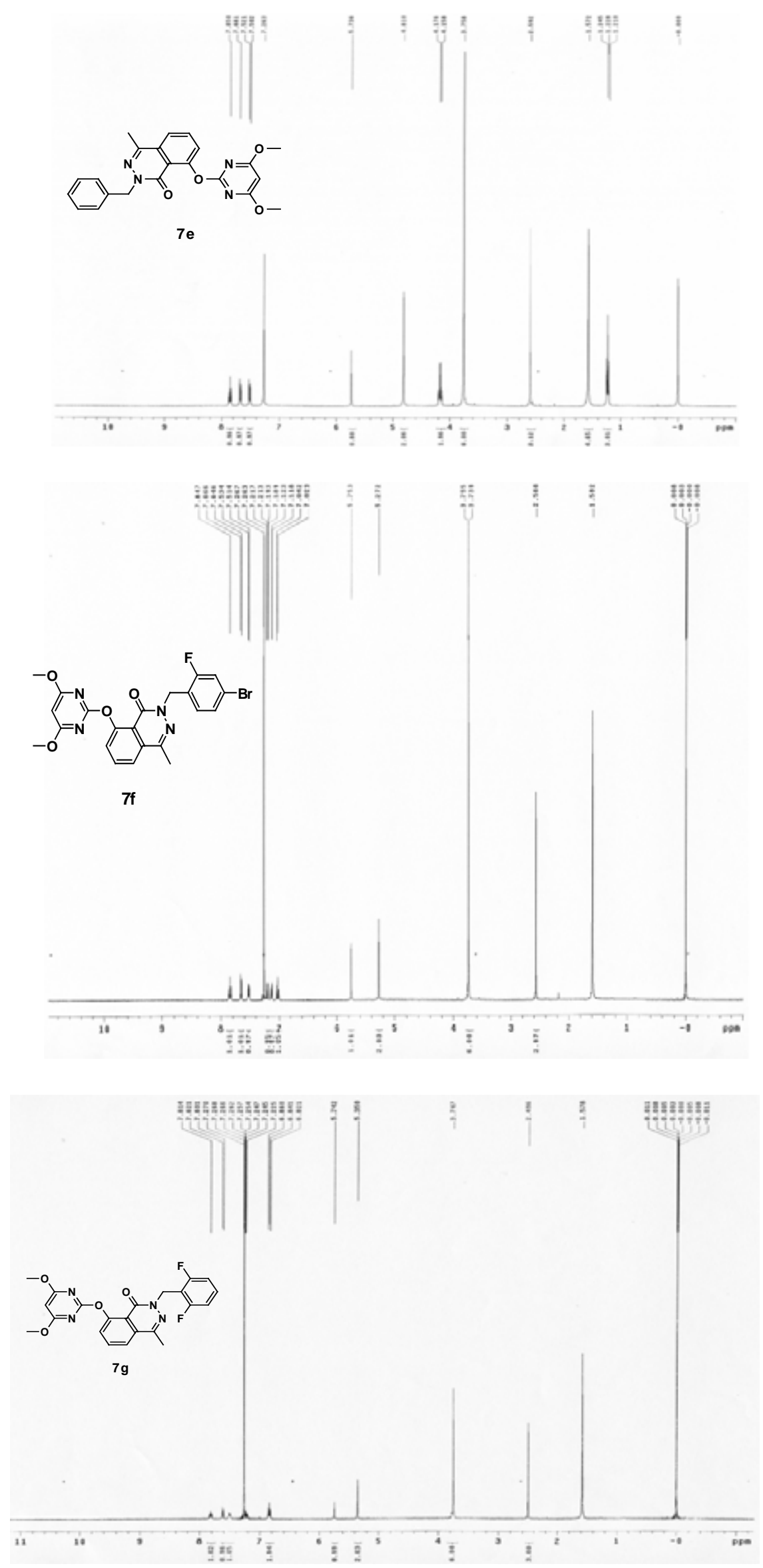

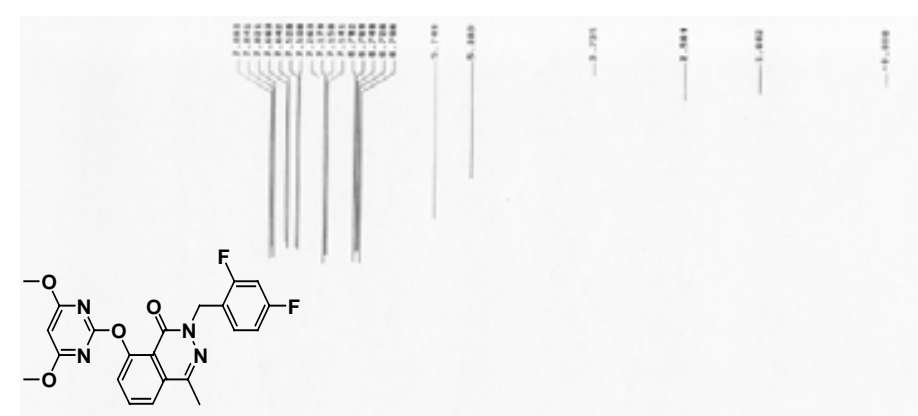

7h
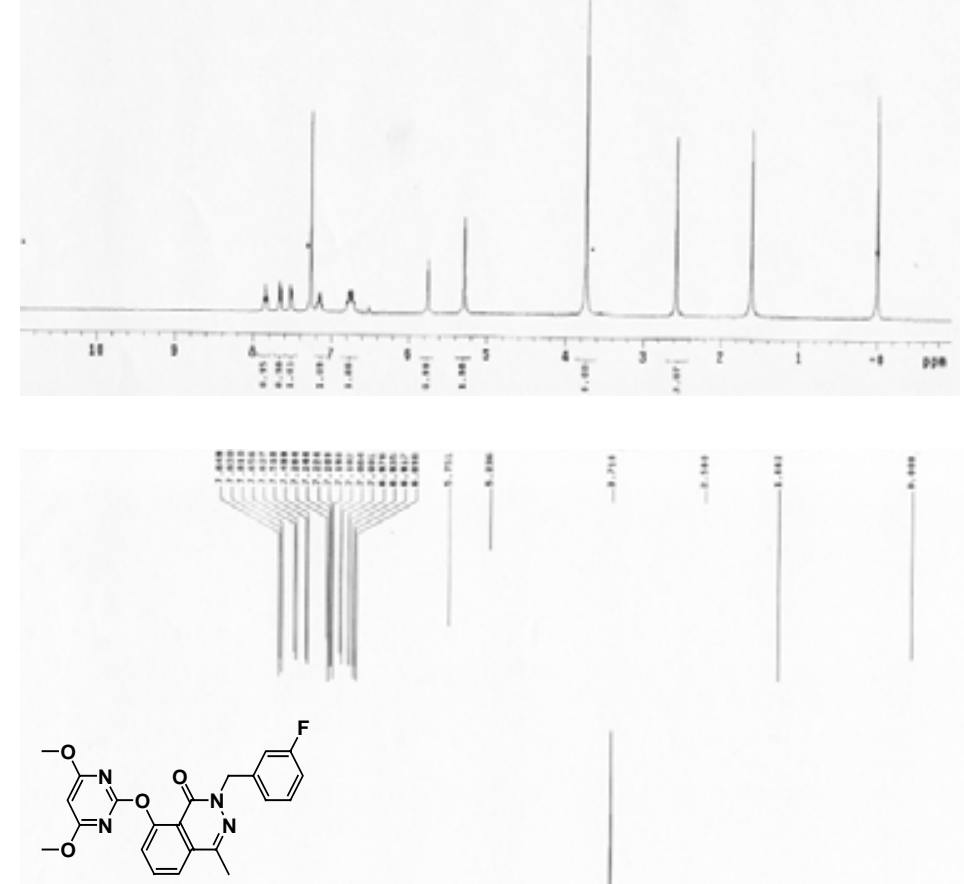

$7 i$
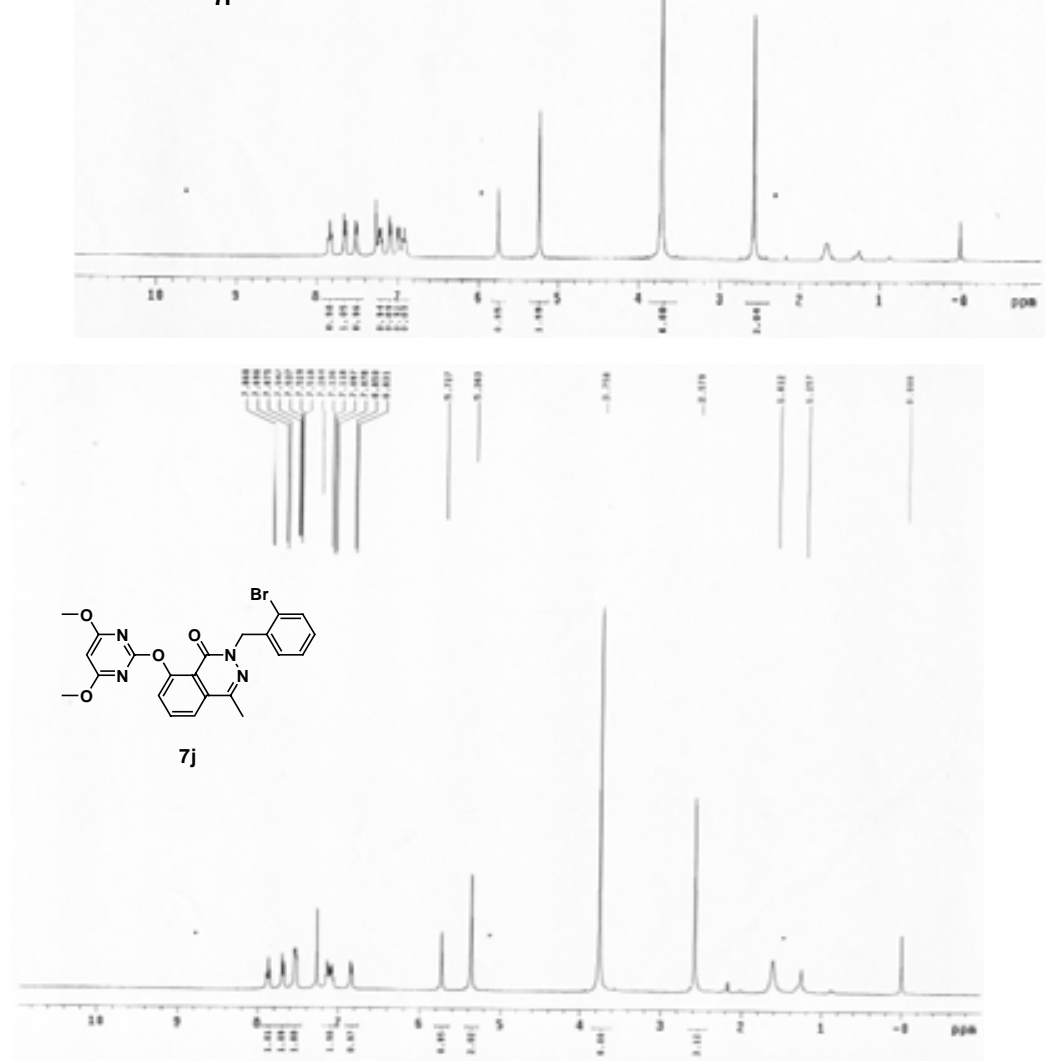

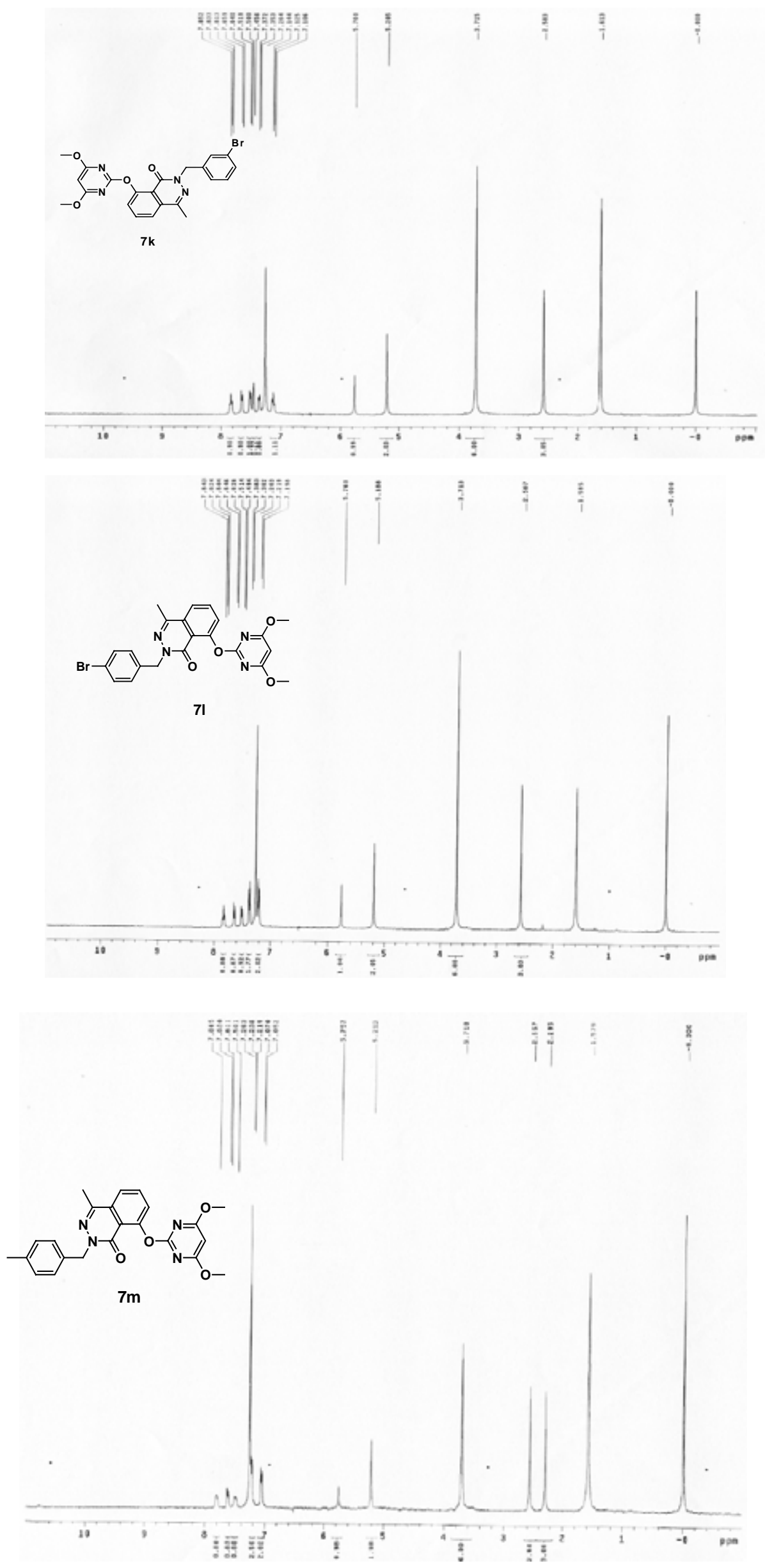

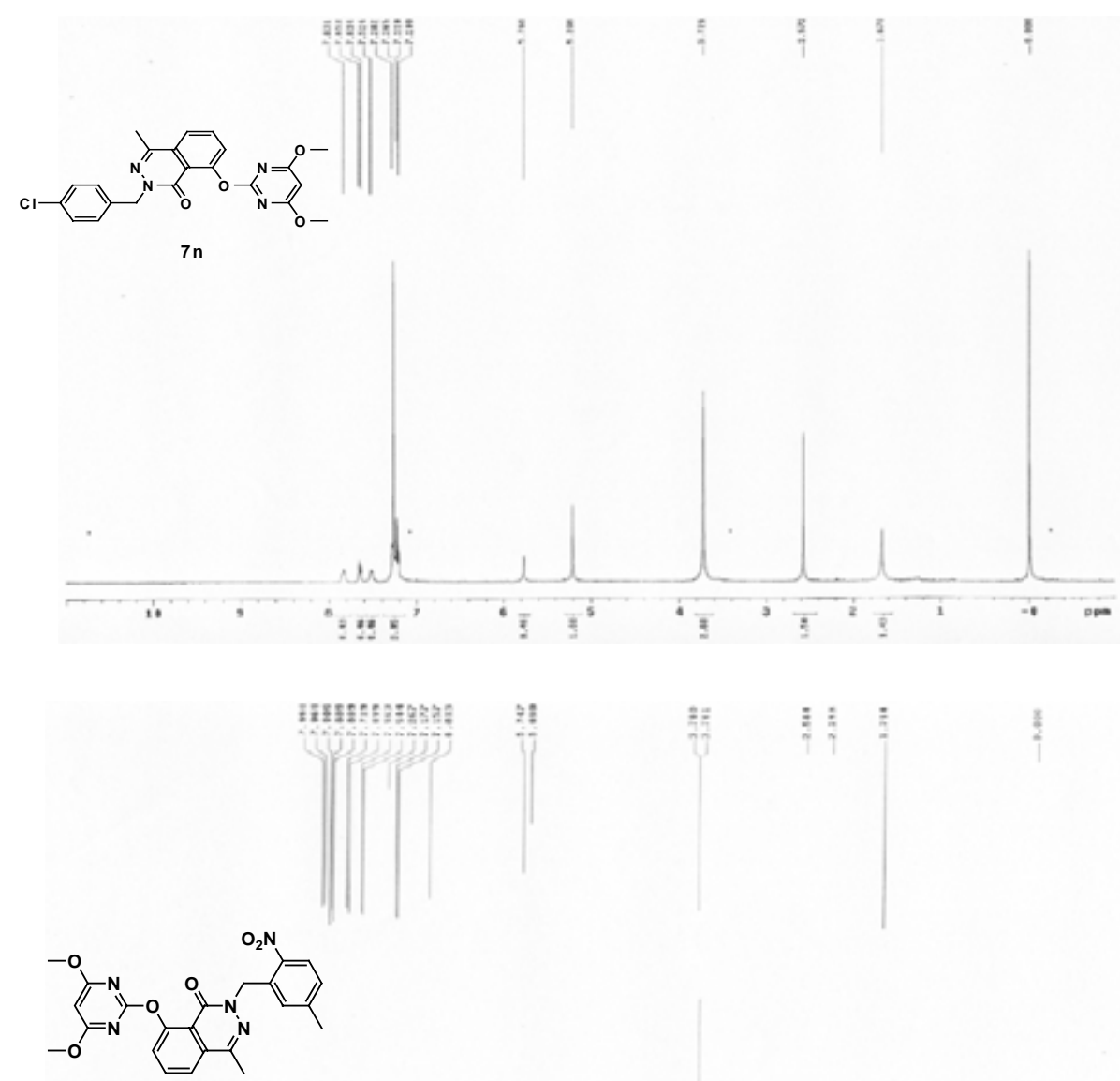

70

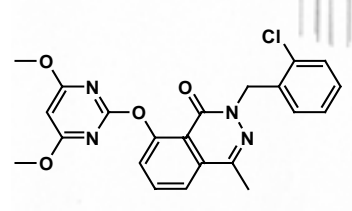

$7 p$

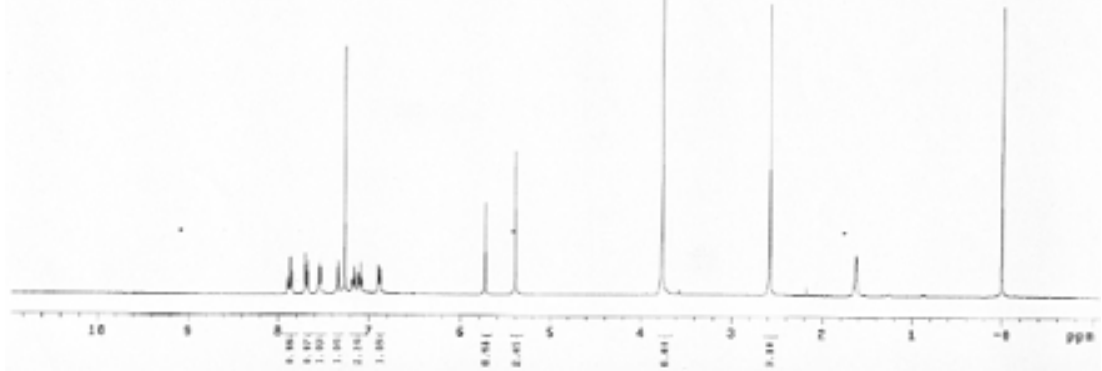



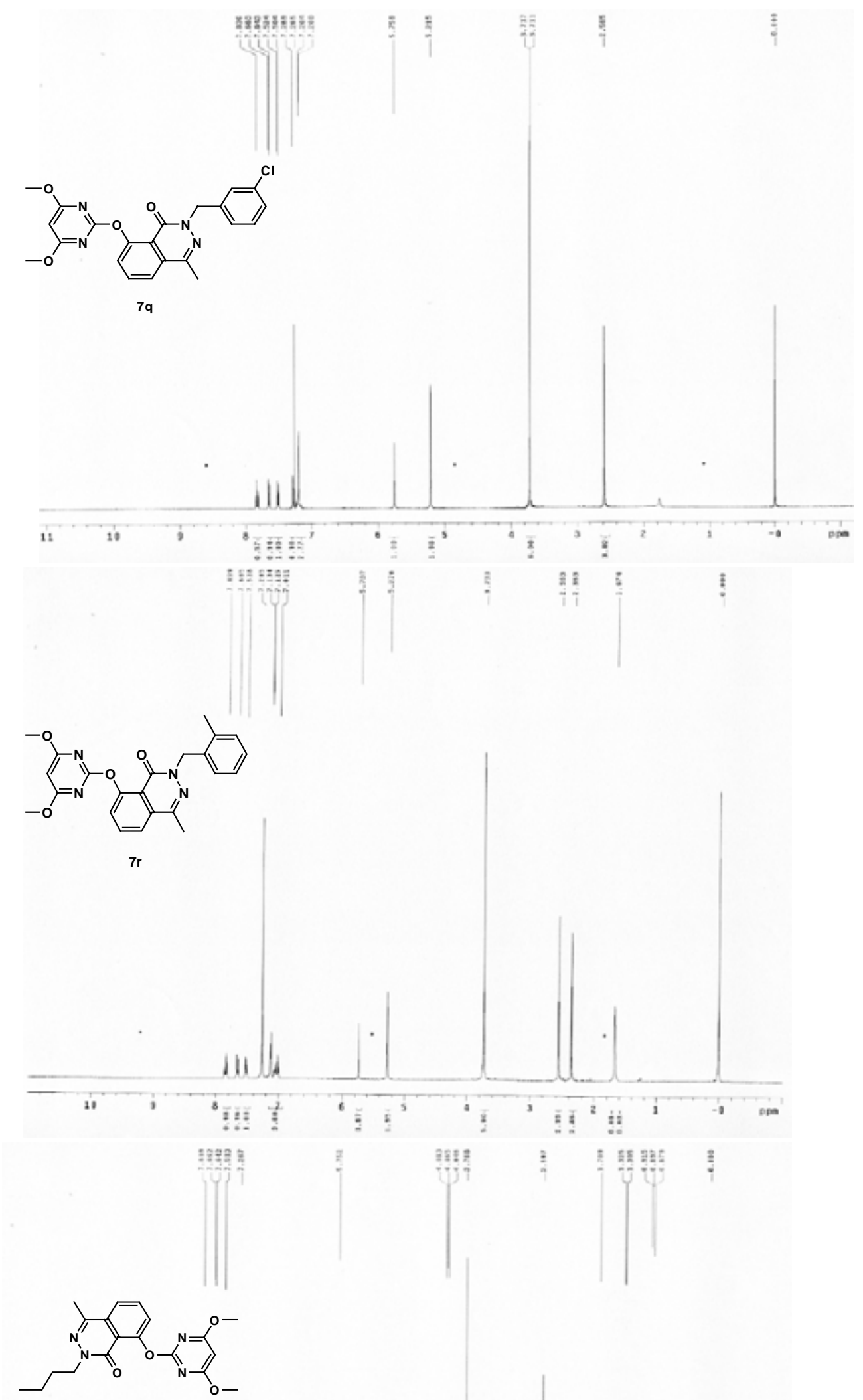

$7 s$ 

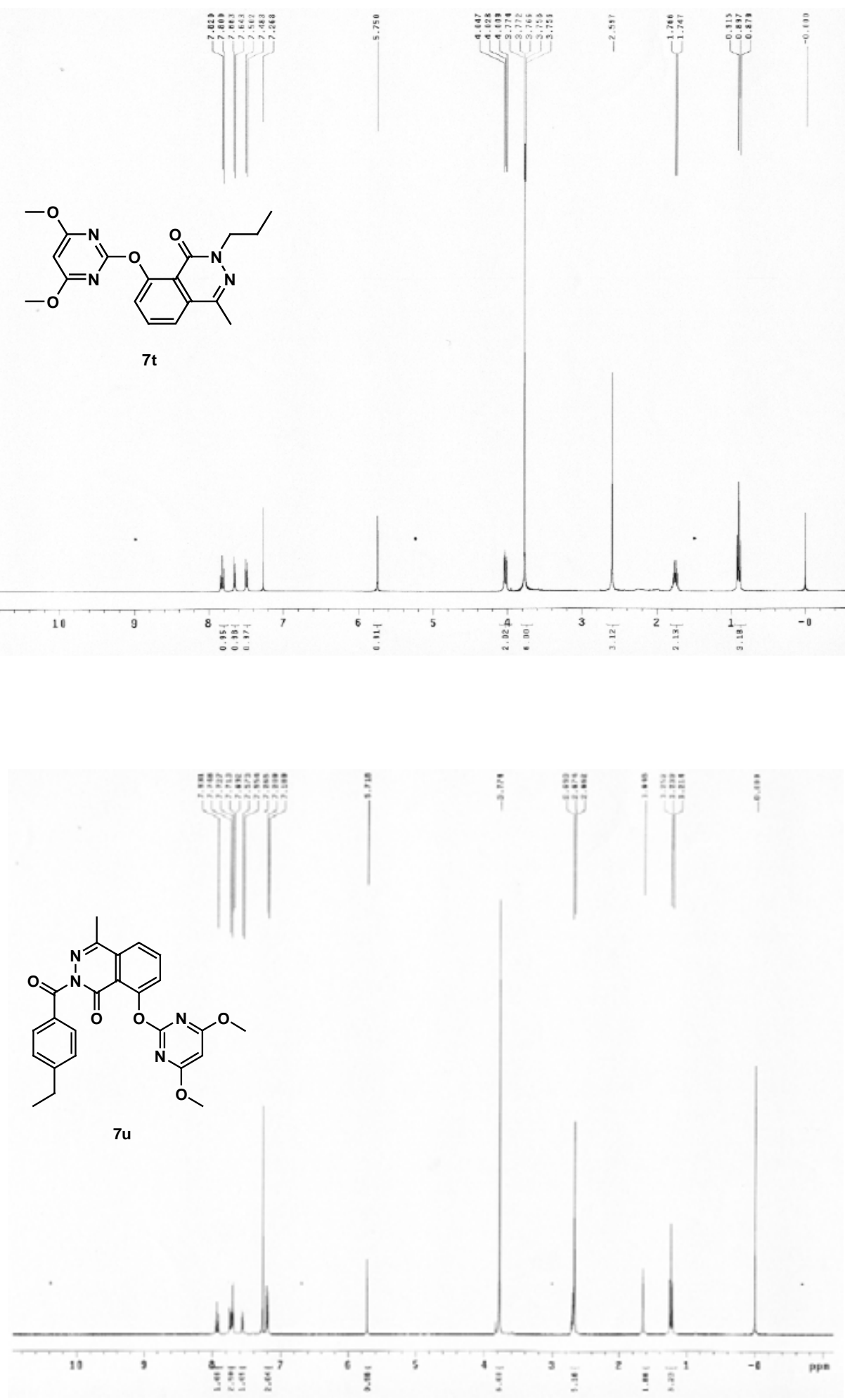

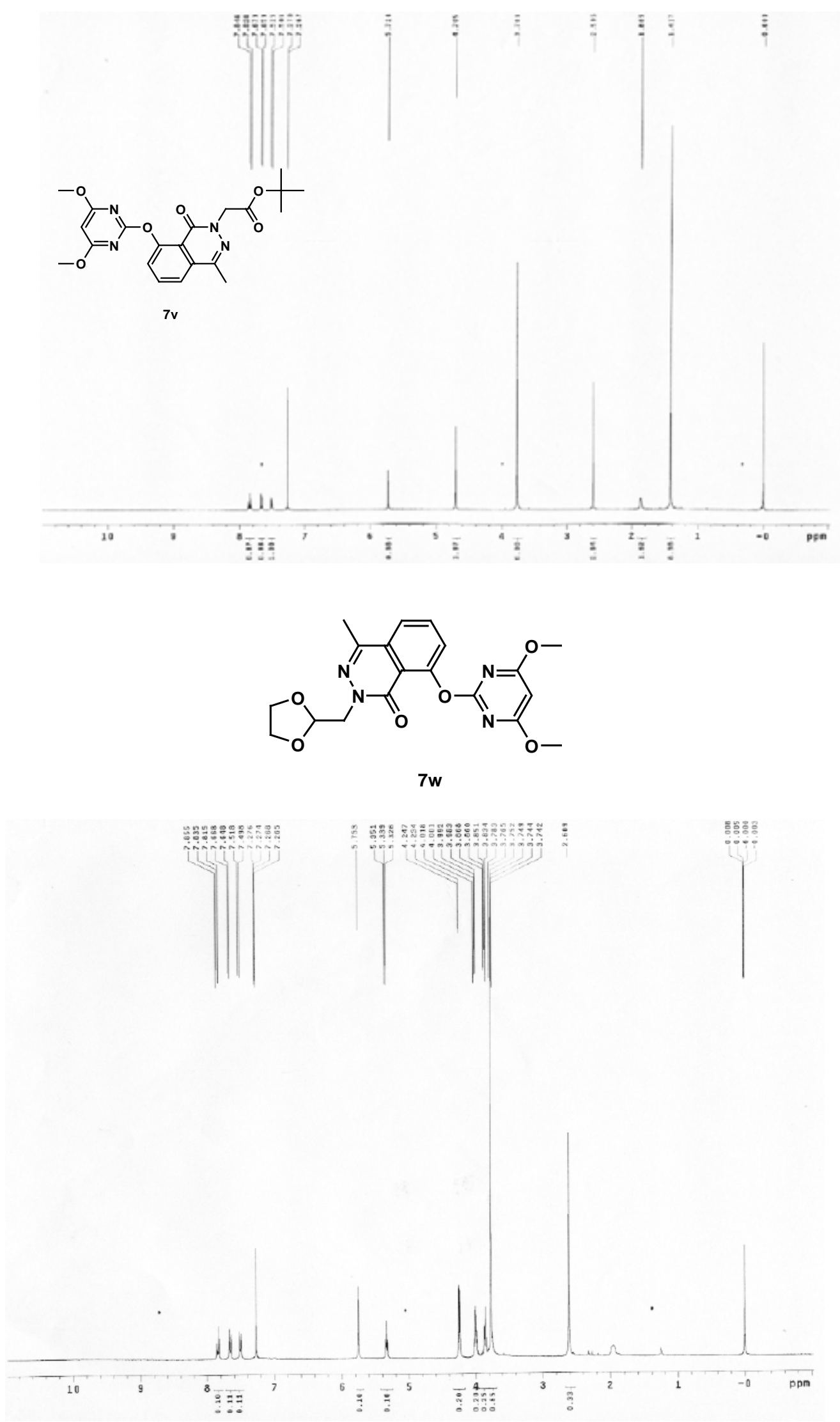